\title{
The role of two-dimensional echocardiography in the detection of potentially embolic intracardiac masses in patients with cerebral ischaemia
}

\author{
RM DONALDSON, RW EMANUEL, CJ EARL \\ From the National Heart Hospital and Cardiothoracic Institute, the Middlesex Hospital, and the \\ National Hospital for Nervous Diseases, London
}

SUMMARY The M-mode and two dimensional echocardiographic data of 62 consecutive cardiac patients referred from neurology centres were analysed retrospectively to establish the use of these techniques in detecting underlying cardiac pathology. All patients had presented initially to a neurologist with transient or permanent focal cerebral or retinal ischaemia, and had been referred for cardiac assessment after neurological investigations failed to establish the underlying cause of the neurological event. Patients were divided into two groups. In 30 patients the referring neurologist had found no evidence of cardiac disease (Group I); in the other 32 patients either heart disease or an arrhythmia had been diagnosed prior to cardiac referral (Group II). One of the patients in Group I had echocardiographic evidence of mitral valve prolapse not detected by the neurologist prior to referral; no cardiac pathology was recognised in the other 29 patients in this group. In seven of the $32(22 \%)$ patients from Group II, a cardiac mass presumed responsible for the neurological manifestations was demonstrated echocardiographically, and in six of these histological confirmation was obtained following surgery or at necropsy. Two dimensional echocardiography was the only investigation which visualised the intracardiac pathology in four patients. In the remaining three patients, valve vegetations (two cases) and an atrial tumour (one case) were demonstrated by both echocardiographic methods. In patients with either clinical evidence of cardiac disease or an arrhythmia who have experienced one or more episodes of cerebral or retinal ischaemia, the presence of an intracardiac mass is not uncommon. Two dimensional echocardiography was the method of choice for detecting cardiac thrombus but the use of both methods of ultrasound should be considered as complementary techniques in the investigation of these cases. Routine echocardiography is unlikely to be of value in screening patients who have had a cerebrovascular event and who do not have clinical evidence of heart disease or an arrhythmia.

Many disorders can cause cerebral ischaemia with transient or permanent neurological dysfunction and the diagnosis of the underlying pathology may present a difficult problem. The range of cardiac conditions alone which are potentially embolic is extensive; early recognition of these may in some cases lead to appropriate therapy and so avert disastrous neurological complications. The purpose of this article is to show the value of the two dimensional echocardiography (2DE) compared with M-

Address for reprint requests: Dr RM Donaldson, National Heart Hospital, Westmoreland Street, London W1M 8BA, UK.

Accepted 1 July 1981 mode echocardiography in the diagnosis of intracardiac masses which are a common source of emboli and to assess the relevance of echocardiographic screening of patients with cerebrovascular events.

\section{Materials and methods}

The sixty-two patients (aged 17 to 68 , mean age 49 years) were consecutive referrals from neurological centres to the National Heart Hospital. In each case the presenting symptoms were such that the patient had been referred to a neurologist in the first instance. Cardiac assessment included conventional M-mode and two dimensional echocardiography (2DE). Retrospective analysis of these techniques was undertaken to determine their relative 
value in establishing the underlying cardiac pathology in this type of neurological referral. All neurological manifestations were due to focal ischaemic events: 19 had experienced one or more transient cerebral or retinal attacks and 43 were admitted to a neurology service with a completed stroke and cerebral infarction. All were referred after neurological investigation had failed to establish the underlying cause of the focal cerebral ischaemia. Patients with stroke due to intracerebral and subarachnoid haemorrhage were not included in the study.

The patients were divided into two groups: those in whom the referring neurologist found no evidence which suggested associated cardiac disease (Group I), and those in whom either heart disease or cardiac arrhythmia had been suspected or diagnosed prior to cardiac referral (Group II). In 30 patients with no clinical evidence of cardiac disease (Group I), echocardiography after referral revealed mitral valve prolapse in one patient, but in the remainder of the group no other cardiac pathology was demonstrated. In the other 32 patients (Group II) there was clinical evidence of heart disease in 17 (ischaemic heart disease in eight, valvular disease in five, congestive failure in two and cardiomyopathy in two) and 15 were referred because of atrial arrhythmias. None of the patients was hypertensive. Out of the 32 patients with clinical evidence of heart disease (Group II), a potentially embolic intracardiac mass, possibly responsible for the neurological manifestations, was identified by echocardiography in seven patients and in six of these cases histological confirmation was obtained following surgery or at necropsy. In four cases the mass was identified only by two dimensional echocardiography and in one case with an atrial tumour this technique clarified the M-mode findings. In the remaining two cases with valve vegetations associated with infective endocarditis the intracardiac lesion was identified by both echocardiographic techniques, although the mobility, localisation and point of attachment of the intracardiac mass was better visualised by two dimensional echocardiography. Four cases in which $2 \mathrm{DE}$ had an important diagnostic role and in whom histopathological data was available are described in detail.

\section{Echocardiographic principles}

Both M-mode and 2DE studies rely on pulsed-reflected ultrasound to image the heart. An ultrasonic transducer is placed on the chest wall and generates short bursts of sound ( 2 to $5 \mathrm{MHz}$ ), which travel along a finite path through the chest. At each interface between tissues with different densities a portion of the sound is reflected back to the transducer, and the remainder continues on, to be reflected by subsequent echo-producing interfaces. The ultrasonic transducer acts as a sound transmitter for a very short time and functions as the echo receiver for the remainder of the interval between transmitted pulses. The pattern of motion of each interface can be analysed by both $\mathrm{M}$-mode and 2DE; these methods differ in their display of ultrasonic information. The M-mode method provides only a limited ("ice pick") unidimensional view of the heart and spatial orientation of the intracardiac structures cannot readily be appreciated. By providing spatial orientation, 2DE allows lateral as well as axial distances between structures to be appreciated; in addition, the images obtained resemble heart structures.

\section{Echocardiographic methods}

Conventional M-mode echocardiography was performed with standard equipment with strip-chart recording of the ultrasonic data. The cross-sectional $2 \mathrm{DE}$ studies were performed using a mechanical sector scanner (SmithKline Instruments Eko-Sector I) with a scanner probe containing a transducer mechanically driven through a $30^{\circ}$ sector at 30 cycles (60 frames) per second. Gain settings and reject control were optimised to avoid misinterpretation of structures. Cross-sectional images were recorded on videotape; the images were then available for analysis in real-time, slow motion or single frame formats. In the static pictures there was, however, loss of visual integration that normally occurs during dynamic recordings. The standard $2 \mathrm{DE}$ was performed on several cross-sectional imaging planes through the heart, from all available acoustic windows, including the parasternal, subcostal and apical transducer positions.

\section{CASE STUDIES}

\section{Case 1}

A 36-year-old man with intermittent atrial arrhythmias and progressive dyspnoea from non-rheumatic mitral regurgitation suffered transient blindness of his left eye which he described as a "curtain" crossing the visual field. Grey-white material, thought to be emboli, was seen in the retinal vessels by opthalmoscopy shortly after this episode of amaurosis fugax. The CT scan was normal and there was no evidence of arterial occlusion on angiography. No abnormalities were detected in the mitral valve region or in the atrium by routine M-mode echocardiography. However, 2DE demonstrated the presence of a slightly mobile mass in the posterior wall of the atrium (fig 1); in addition there were echoes suggestive of a thrombus formation near the posterior leaflet of the mitral valve. Six months later the mitral valve was repaired: the presence of a thrombus $(2 \times 1 \mathrm{~cm})$ in the atrium and in the area of attachment of the valve was confirmed. Histologically, the thrombus had areas of organisation at the base, although other sections were of more recent age.

\section{Case 2}

A 45-year-old man had a right hemiparesis (predominantly affecting the lower limb) six months after an anterior myocardial infarction. There was additional sphincter involvement, but no loss of consciousness. The CSF study was normal and the cerebral infarction revealed by CT was considered most likely due to distal embolic occlusion of the anterior cerebral artery. He made a good neurological recovery. However, over the next year he required cardiac assessment because of disabling angina. At that stage the 2DE demonstrated apical wall motion abnormality and the presence of multiple dense layered echoes moving within the ventricular cavity adjacent to the lateral wall of the ventricular apex suggested endocardial 


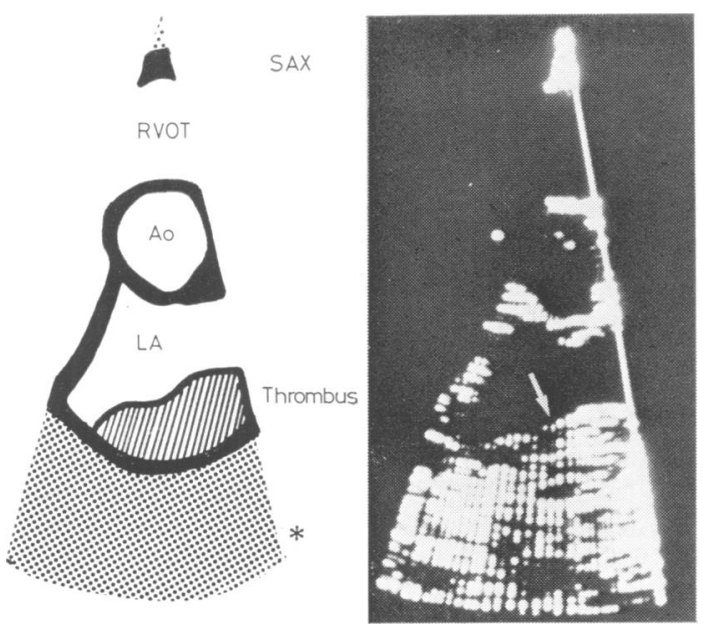

Fig 1 Cross-sectional (short axis-SAX) view of the left atrium $(L A)$ at the level of the aorta $(A o)$ demonstrating the presence of left atrial $(L A)$ thrombus (arrow). RVOT: Right Ventricular Outflow Tract.

thrombus (fig 2a), best visualised in the apical views. Cardiac catheterisation was followed by aneurysmectomy and saphenous vein bypass grafting. Histologically the thrombus demonstrated various stages of organisation and was attached to the aneurysmal myocardium which consisted predominantly of collagen fibres (fig $2 \mathrm{~b}$ ).

\section{Case 3}

A 32-year-old woman with persistent blood and bone marrow eosinophilia (for which no underlying aetiology could be established) had progressive organ system dysfunction attributable to tissue invasion by eosinophils. Initially, clinical and laboratory investigations showed involvement of the lungs, liver, spleen and heart. There was congestive cardiac failure with auscultatory evidence of mitral regurgitation. The heart failure responded favourably to digitalis and diuretics. She was admitted with progressive neurological signs which included right sided spastic hemiplegia affecting upper and lower limbs, sensory defects (loss of sense of passive movements) and dysphasia. The CSF obtained by lumbar puncture was clear, with a raised $\left(20 / \mathrm{mm}^{3}\right)$ white cell count and normal protein level. Computed tomography showed a cerebral infarct in the left hemisphere. There was no evidence of diffuse central nervous system involvement or peripheral neuropathy and the symptoms were considered most likely to have been due to the thromboembolic occlusion of the middle cerebral artery seen on angiography. The echocardiographic study visualised increased wall thickness and acoustic density and the additional intracavitary multiple layered echoes contiguous with the ventricular wall suggested mural thrombi (fig 3 ). In spite of therapy which included anti-hypereosinophilic drugs (corticosteroids and cytotoxic agents), and anti-platelet agents (aspirin and dipyridamole), the patient died six

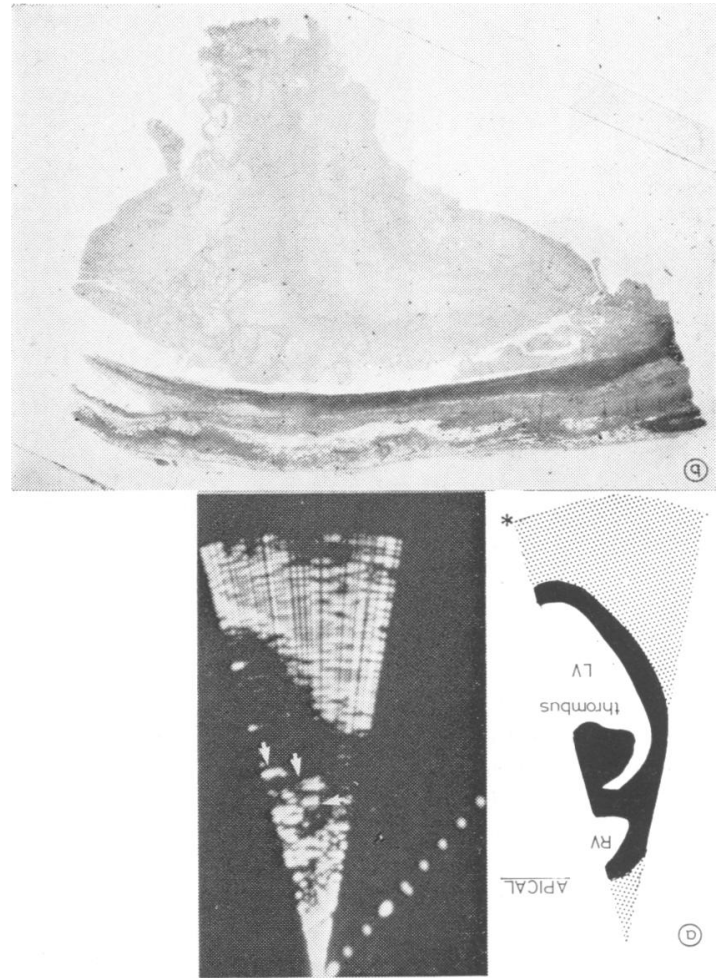

Fig 2 Ventricular thrombus. (A) Apical view showing intracavitary echoes (small arrows) contiguous with the left ventricular ( $L V)$ wall, denser than the adjacent myocardium. Note central area of sonolucency. (B) Left ventricular aneurysmal wall and superimposed thrombus, with evidence of organisation at the base. Miller's elastic Van Gieson × 4 .

months after the 2DE study. Necropsy revealed eosinophilic infiltration in the myocardium and confirmed the presence of fresh and organised mural thrombi $(2 \times 2 \mathrm{~cm})$ in the left ventricular outflow regions, characteristic Löefflers endocarditis parietalis fibroplastica. No vascular lesions in the carotid artery territory were present, but brain infarction was confirmed histopathologically in the region of the middle cerebral artery.

\section{Case 4}

A 27-year-old male patient underwent echocardiographic study following a cerebral infarction leading to right sided motor and sensory defects. He had atrial fibrillation with clinical features of mixed mitral valve disease and the possibility of thromboembolism had been raised at the initial examination. The CSF was not blood stained and an evolving infarction was seen on serial CT studies. There was evidence of mitral stenosis on the M-mode echocardiogram, with cusp thickening and anterior movement of the posterior leaflet. Multiple extra echoes were visualised beneath the anterior mitral cusp during 


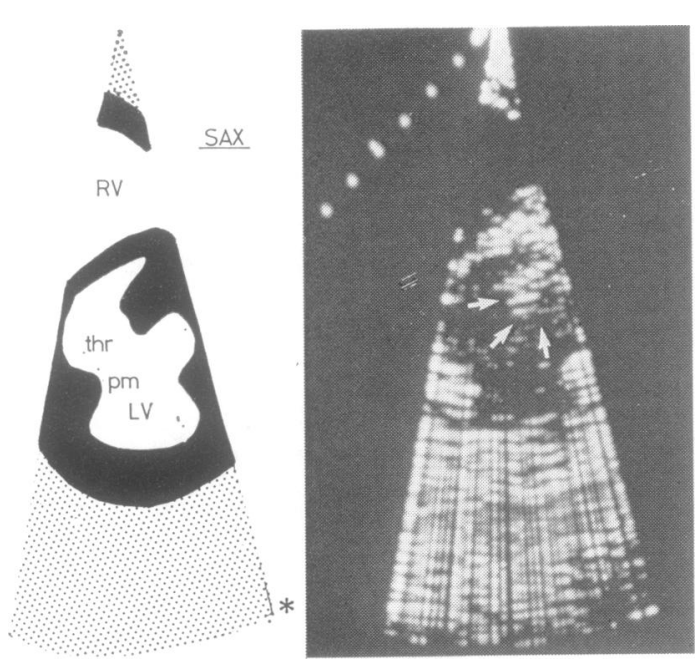

Fig 3 Short axis (SAX) view of the left ventricle ( $L V)$ at papillary muscle (PM) level, showing increased wall thickness and acoustic density (depicted in black) and the presence of thrombus (THR) (arrows) in the patient with hypereosinophilic syndrome. LV: Left Ventricle; $R V$ : Right Ventricle.

diastole and additional systolic echoes behind the posterior wall of the aorta suggested the presence of a mobile atrial mass (fig 4a). The 2DE permitted further visualisation of the mass: a pedunculated tumour was demonstrated and shown to be attached to the atrial septum. It moved freely within the atrium throughout the cardiac cycle, prolapsing into the reduced mitral valve orifice during diastole (figs $4 \mathrm{~b}$ and $4 \mathrm{c}$ ). The diagnosis of atrial myxoma and rheumatic mitral valve disease was confirmed at surgery and subsequently histologically.

\section{Discussion}

The role of $\mathrm{M}$-mode echocardiography in the detection of cardiac masses is well established. 12 Recent developments in 2DE techniques enables direct visualisation of nearly all areas of the heart and permits the simultaneous study of its chambers. ${ }^{3} 4$ Two dimensional echocardiography displays spatial characteristics of the image by rapidly steering the ultrasound beam back and forth through a given field of view, and these images have added new and more precise data to that obtained by M-mode echocardiography. Because these images anatomically resemble the heart, $2 \mathrm{DE}$ is capable of visualising the cardiac masses rather than giving indirect evidence as does the conventional M-mode technique. The potential for spurious echoes to appear within the heart in M-mode recordings is also well recognised; in the $2 \mathrm{DE}$ studies, in which views are obtained from various locations around the thorax, the margin for error is reduced as spurious echoes are seen in only a single view, whereas intracardiac masses could be consistently visualised. In the selected population studied echocardiographically, there was an $11 \%$ overall incidence of an associated intracardiac mass presumed responsible for the neurological symptoms. The M-mode technique proved to be of value in the localisation of vegetations and suggested the presence of the atrial tumour (Case 4), but was not reliable in the demonstration of other intracardiac pathology. The 2DE appearances of an intracardiac mass in our experience have been similar whether the mass consisted of a thrombus, vegetation, tumour or calcific deposit on a cardiac valve. The location, size, origin, shape and motion of the mass was important in determining the nature of the lesion and could readily be appreciated from the images.

A cerebral embolus is a much dreaded complication of cardiac disease as it often occurs unexpectedly in patients whose symptoms are few and whose prognosis is otherwise good. Emboli from within the heart are well recognised as a frequent cause of stroke $; 5$ this appears particularly relevant in young adults, where embolisation of thrombotic material from the heart is a relatively frequent cause of neurological symptoms. 6 ?

The diagnostic difficulties encountered in the use of M-mode echocardiography are well known in the presence of thrombi in contact with the atrial or ventricular wall. ${ }^{2}$ Using the multiple imaging planes, 2DE can look into many portions of the left atrial cavity and demonstrate thrombus formation (fig 1) which is secondary to abnormalities of atrial size, rhythm or wall surface properties that occur alone or in combination with mitral valve pathology. Because it records larger portions of the atrium and permits visualisation of the dynamic movement of the mass, 2DE facilitates the interpretation of vague M-mode findings, and is the superior technique in the detection of atrial thrombus. ${ }^{2} 8$ Although relatively recent thrombi can be visualised, some degree of organisation is probably required for their accurate identification, and thrombi usually have to be larger than $2 \mathrm{~cm}$ in diameter. ${ }^{9}$ Cerebral ischaemic lesions in young patients with mitral valve prolapse can result from thromboembolism;10 2DE has revealed the presence of thrombi in the atrium and attached to prolapsing valves in some of these patients presenting with transient ischaemic attacks or stroke, ${ }^{11}$ as in the young patient reported in Case 1. In patients with rheumatic heart disease $2 D E$ can detect the development of a ball-valve thrombus in the left atrial cavity, which can cause fluctuating changes in the degree of obstruction, sornetimes with syncope or sudden death. 

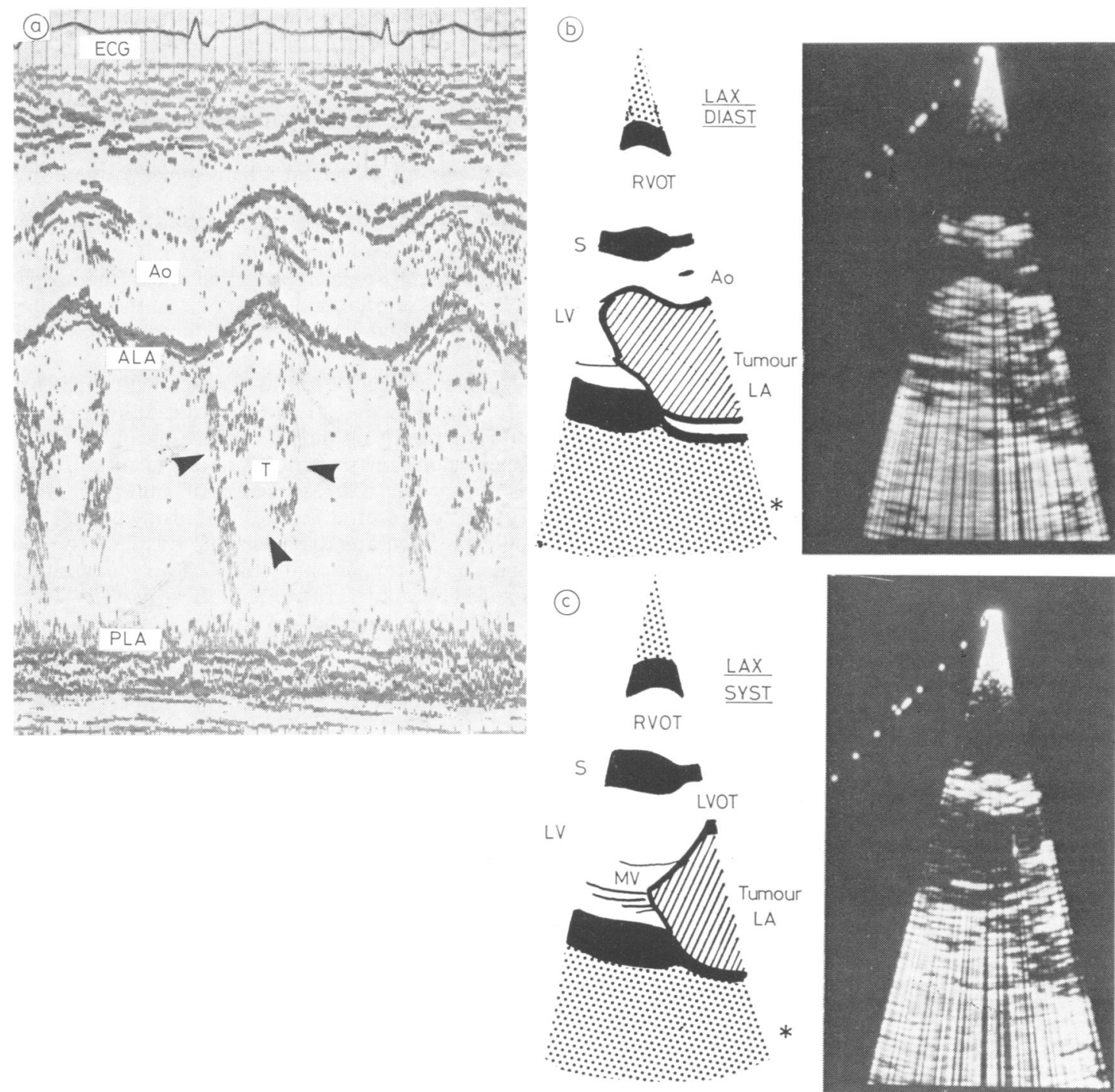

Fig 4 (A) Left atrial myxoma: M-mode echocardiogram. The tumour echoes (T) fill the left atrium during ventricular systole. ALA: Anterior Left Atrial Wall; PLA: Posterior Left Atrial Wall. (B) $2 D E$ recording. Left atrial myxoma (tumour LA) prolapsing into the reduced mitral valve orifice during diastole (diast) (Long axis scan-LAX). (C) Propelled back into the atrium during ventricular systole (syst). Other legends as in previous figs.

In patients with dilated (congestive) cardiomyopathy, transmural infarction or ventricular aneurysm, stroke can result from transient arrhythmias and hypotensive episodes; however intracardiac thrombus formation due to stasis of blood or endocardial injury is relatively common in these conditions and can be a source of systemic emboli. Definitive antemortem diagnosis of ventricular thrombus is difficult; its presence is usually clinically apparent if an embolic event occurs or if detected by ventriculography. The latter, however, has a low sensitivity. ${ }^{12}$ Likewise, M-mode echocardiography, with its inability to image detail at the ventricular apex (the most common location of thrombus) has failed as a useful tool for visualisation of ventricular thrombus except in isolated rare instances. ${ }^{13-16}$ With $2 D E$ and the use of multiple cross-sectional planes, optimal assessment of the left ventricular apex is possible. 
Prospective studies are required to evaluate the diagnostic accuracy of this method. A protruding intracavitary mass of echoes contiguous with the ventricular wall and denser than the adjacent myocardium associated with an akinetic or aneurysmal myocardial segment (particularly apical) can, however, be assumed to represent mural thrombus in the obvious clinical setting as in Case 2. Similar findings were documented in a second patient in Group II who presented with focal neurological disturbances 3 weeks after a large anterior infarction, but in whom histopathological confirmation was not available. The ease with which left ventricular wall motion can be visualised and the potential for detection of mural thrombus suggest that 2DE may be useful in the selection of patients at risk from embolisation who would benefit from anticoagulant therapy. ${ }^{17}$ There are however no specific echocardiographic patterns for thrombus, and other possibilities such as normal apical structures or pathological echoes due to tumours, masses of infective material or technical artifacts should be considered in the differential diagnosis. The irregular shape and appearance of recent ventricular thrombi in patients with coronary artery disease (fig 2) appears to differ from the larger circumscribed appearance of chronic thrombi in patients with congestive cardiomyopathy. ${ }^{18}$ Technical problems (particularly inappropriate gain attenuation settings and resolution problems) and the acoustical similarity of small thrombi to the surrounding blood or endocardium hinder the sensitivity of this method. ${ }^{19}$

Cardiovascular involvement occurring in $80 \%$ of patients with the hypereosinophilic syndrome ${ }^{20}$ includes infiltrative cardiomyopathy, ventricular wall thickening, mitral regurgitation and mural thrombi. ${ }^{21}$ Localised accretions of echo-dense material in the posterior left ventricular wall beneath the mitral valve and apical intracavitary masses contiguous with the ventricular wall and consistent with thrombus as seen in Case 3 (fig 3) have been documented and confirmed histologically in patients with the thrombotic stage of cardiac involvement, which is characterised by the presence of acute and organising mural thrombi in the ventricular inflow regions. ${ }^{22}$ The echocardiographic pattern of infiltration is non-specific and should be distinguished from other causes of increased wall thickness and acoustic density. The frequency of embolic events in this condition is about $6-10 \%$; early recognition of mural thrombi should lead to earlier anticoagulation of those patients at risk from emboli.

Early diagnosis of myxomas is essential because of their obstructive, systemic and embolic complications; the last of these occurring in $30 \%$ of the cases.
The most frequent location of myxomas is in the left atrium: typically these benign tumours are pedunculated, attached to the septum and move freely throughout the cardiac cycle, prolapsing into the mitral valve orifice during diastole (fig $4 \mathrm{~b}$ ), and being propelled back into the left atrium during ventricular systole (fig 4c). A left atrial myxoma is, therefore, potentially recognisable, not only as an intracavitary mass, but also by virtue of its point of attachment and the motion exhibited. The diagnosis of left atrial myxomas represented one of the earliest applications of echocardiography, and the reliability of these methods of screening tumours has been reported. ${ }^{23-25}$

The association of mitral stenosis with thickened cusps (as in Case 4) may occasionally produce confusing results on the M-mode study and bands of echoes behind the posterior wall of the aorta (fig 4a) are nonspecific. The presence of other tumours, thrombi, technical difficulties and artifacts may also hinder the accurate diagnosis of myxomas by the standard unidimensional study. Two dimensional echocardiography is of value in those cases in which M-mode imaging produces equivocal results. ${ }^{2}$

\section{Conclusions}

Two dimensional echocardiography was the only investigation which visualised the intracardiac pathology in four patients. In the remaining three patients, valve vegetations (two cases) and an atrial tumour (one case) were demonstrated by both echocardiographic methods. In selected patients with either clinical evidence of cardiac disease or an arrhythmia who have experienced one or more episodes of cerebral or retinal ischaemia, the presence of an intracardiac mass is not uncommon, and the complementary use of both methods of ultrasound should aid clinical management. Two dimensional echocardiography is however the method of choice for detecting cardiac thrombus and should be undertaken in all such cases.

In patients with neurological symptoms without clinical manifestation of heart disease, this technique failed to provide evidence of underlying cardiac pathology. Routine echocardiographic screening is unlikely to be of value in investigation of patients who have had a cerebrovascular event, and we suggest that such screening be reserved for selected patients.

We are grateful to Drs $\mathbf{R}$ Foale and $\mathbf{M}$ Ballester for their help with the echocardiographic recordings. The detailed histopathological data was provided by Dr E Olsen. 


\section{References}

${ }^{1}$ Popp AL. Echocardiographic assessment of cardiac disease. Circulation 1976;54:538-52.

2 Feigenbaum H. Echocardiography, 3rded. Philadelphia: Lea \& Febinger, 1981.

${ }^{3}$ Kisslo J, VonRamm OT, Thurstone FL. Cardiac imaging using a phased-array ultrasound system. II : Clinical techniques and applications. Circulation 1976;53:262-7.

* Tajik AJ, Seward JB, Hagler DJ, Mair DD, Lie JT. Two dimensional real-time ultrasonic imaging of the heart and great vessels. Technique, image orientation, structure identification and validation. Mayo Clin Proc 1978;53:271-303.

${ }^{5}$ Whisnant JP. A population study of stroke and TIA: Rochester, Minnesota. In: Gillingham FJ, Maudsley C, Williams AE, eds. Stroke. London: Churchill Livingstone 1976:20-39.

${ }^{6}$ Grindal AB, Cohen RJ, Saul RF, Taylor JR. Cerebral infarction in young adults. Stroke 1978;9:39-42.

7 Wilson LA, Warlow CP, Ross Russell RW. Cardiovascular disease in patients with retinal arterial occlusion. Lancet 1979; : $292-4$.

${ }^{8}$ Schweizer P, Bardos P, Erbel R et al. Detection of left atrial thrombi by echocardiography. Brit Heart $J$ $1981 ; 45: 148-56$.

${ }^{9}$ Ports TA, Cogan J, Schiller NB, Rappaport E. Echocardiography of left ventricular masses. Circulation 1978;58:528-36.

${ }^{10}$ Barnett KJM, Boughner DR, Taylor DW, Cooper DE, Kustuk WJ, Nichol PM. Further evidence relating mitral valve prolapse to cerebral ischaemic events. New Engl J Med 1980;302:139-44.

11 Rothbard RL, Nanda NC, Fleck G, Heinle RA. Mitral valve prolapse and stroke: detection of potential emboli by real time two dimensional echocardiography. Circulation 1979;60(Suppl II):99.

${ }^{12}$ Reeder GS, Lengyel M, Tajik AJ et al. Mural thrombus in left ventricular aneurysm. Mayo Clin Proc 1981; 56:77-81.

${ }^{13}$ Horgan JH, Shiel FO, Goodman AC. Demonstration of left ventricular thrombus by conventional echocardiography. $J C U$ 1976;4:287-8.
14 DeJoseph RL, Shiroff RA, Levenson LW, Martin CE, Zelis RF. Echocardiographic diagnosis of intraventricular clot. Chest 1977;71:417-9.

${ }^{15}$ Kramer NE, Rathod R, Chawla KK, Patel R, Towne WD. Echocardiographic diagnosis of left ventricular mural thrombi occurring in cardiomyopathy. Am Heart J 1978;96:381-3.

${ }^{16}$ Van den Bos AA, Vletter WB, Hagemeijer F. Progressive development of a left ventricular thrombus: detection and evolution studied with echocardiographic techniques. Chest 1978;74:307-9.

17 Veterans Administration Hospital Trial. Anticoagulants in acute myocardial infarction. JAMA 1973; 225:724-9.

${ }^{18}$ DeMaria AN, Neumann A, Bommer W et al. Left ventricular thrombi identified by cross-sectional echocardiography. Ann Intern Med 1979;90:14-8.

${ }^{19}$ Asinger RW, Mikell FL, Sharma B, Hodges M. Observations on detecting left ventricular thrombus with two dimensional echocardiography: emphasis on avoidance of false positive diagnosis. Am J Cardiol 1981;47:145-56.

${ }^{20}$ Chusid MJ, Dale DC, West BC, Wolff SM. The hypereosinophilic syndrome: analysis of 14 cases with review of the literature. Medicine (Baltimore) 1975;54:1-27.

${ }^{21}$ Parrillo JE, Borer JS, Henry WL, Wolff SM, Fauci AS. The cardiovascular manifestations of the hypereosinophilic syndrome. Prospective study of 26 patients with review of the literature. Am J Med 1979; 67:572-82.

${ }^{22}$ Hall SW Jr, Theologides A, From AHL et al. Hypereosinophilic syndrome with biventricular involvement. Circulation 1977;55:217-22.

${ }^{23}$ Finnegan RE, Harrison DC. Diagnosis of left atrial myxoma by echocardiography. New Engl J Med 1970; 282:1022-3.

${ }^{24}$ DeMaria AN, Visnamara LA, Miller RA et al. Unusual echocardiographic manifestations of right and left heart myxomas. Am J Med 1975;59:713-20.

${ }^{25}$ Lappe DL, Bulkley BH, Weiss JL. Two dimensional echocardiographic diagnosis of left atrial myxoma. Chest 1978;74:55-8. 